\title{
Steuern statt verwalten
}

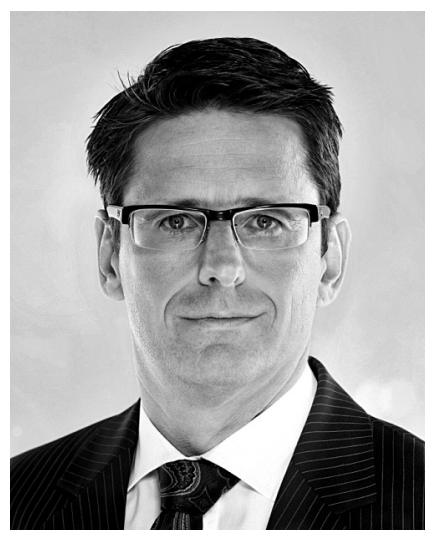

VON ATTILA NAGY

Attila Nagy ist geschäftsführender Partner der rosenbaum nagy unternehmensberatung $\mathrm{GmbH}$ in Köln.

www.rosenbaum-nagy.de

\author{
"Bürokratiekosten« können durch eine funktionierende \\ und schlanke Verwaltung zwar eingespart \\ werden, entscheidender sind jedoch Aspekte einer \\ effizienten und ständig nachjustierten Steuerung \\ einer Organisation oder eines Unternehmens.
}

Verwaltungsaufgaben und erst recht Verwaltungseinheiten haben in allen Bereichen des Lebens ein Imageproblem. Dies ist in Teilen sicherlich begründet, denn einerseits müssen sie sich im Gegensatz zu den produktiven Bereichen häufig nicht unmittelbar am Markt beweisen, so dass sich ineffiziente Strukturen und Prozesse hier länger erhalten können. Andererseits entwickeln sie - einmal etabliert - einen Selbsterhaltungstrieb, bis hin zur Entwicklung von Wachstumstendenzen. Zugleich wird aber das Fehlen von Kontrolle sofort kritisch angeprangert, sobald sich innerhalb von Organisationen gewisse Fehlentwicklungen zeigen oder beispielsweise anlässlich von öffentlichkeitswirksamen Fehlern in der Leistungserbringung.

Diese Beispiele zeigen, dass in vielen Bereichen Verwaltungsaufgaben und der mit ihnen verbundene Aufwand unumgänglich sind, denn sie ermöglichen eine Steuerung und Kontrolle der zu erbringenden Leistungen sowohl für die internen Entscheidungsträger als auch für externe Anspruchsgruppen. Dennoch stellt sich die Frage, wie das richtige Maß zu finden ist.

Aus dem Blickwinkel eines sozialen Unternehmens ist ein möglichst geringer Umfang an extern vorgegebenen Verwaltungsaufgaben wünschenswert, denn dies reduziert den zu leistenden Aufwand. In diesem Zusammenhang kann ein einzelnes Unternehmen jedoch im Wesentlichen nur beeinflussen, wie den externen Anforderungen organisatorisch zu begegnen ist. Bei der Festlegung der internen Anforderungen an die Verwaltung sind die Freiräume erheblich größer und bei diesen spielen Kosten-Nutzen-Überlegungen eine größere Rolle, wobei auch Risikoaspekte berücksichtigt werden müssen.

Eine generelle Aussage darüber, wie viel Bürokratie eine Organisation braucht oder wie eine optimale Verwaltung auf allen Ebenen aufgestellt zu sein hat, ist aufgrund der Heterogenität der Organisationen und der Vielzahl von Variablen nicht möglich. So können wir aus unserer Erfahrung heraus sagen, dass kleine Organisationen im Bereich der Zentralverwaltung (Personal, Rechnungswesen) tendenziell weniger effizient sind als größere Organisationen.

Dies gilt jedoch nicht zwingend für jeden Einzelfall und erst recht nicht, wenn wir einen erweiterten Begriff von »Bürokratiekosten « einschließlich verschiedener Stabs- und Führungsfunktionen sowie des Ressourcenbedarfs bei den Führungskräften der operativen Einheiten mitbetrachten. Denn der Organisationsaufwand wächst mit der Komplexität und durch die Größe der Organisationen steigt die Komplexität zumeist erheblich an. Diese allgemeinen Tendenzen können auf den wirtschaftlichen Erfolg von wachsenden Organisationen gewisse Auswirkungen haben (vgl. Abb. 1).

Zur Ermittlung der Gesamtkosten je Einheit können zunächst die »Produktionskosten « betrachtet werden (1), die in der Regel mit einer steigenden Unternehmensgröße je produzierte Einheit sinken. In einem vereinfachten Modell kommen hierzu noch »Bürokratiekosten « in einem erweiterten Sinne, das heißt unter Einbeziehung von sämtlichen Verwaltungs-, Leitungs- und Koordinationsaufgaben, einschließlich der gebundenen Ressour- 


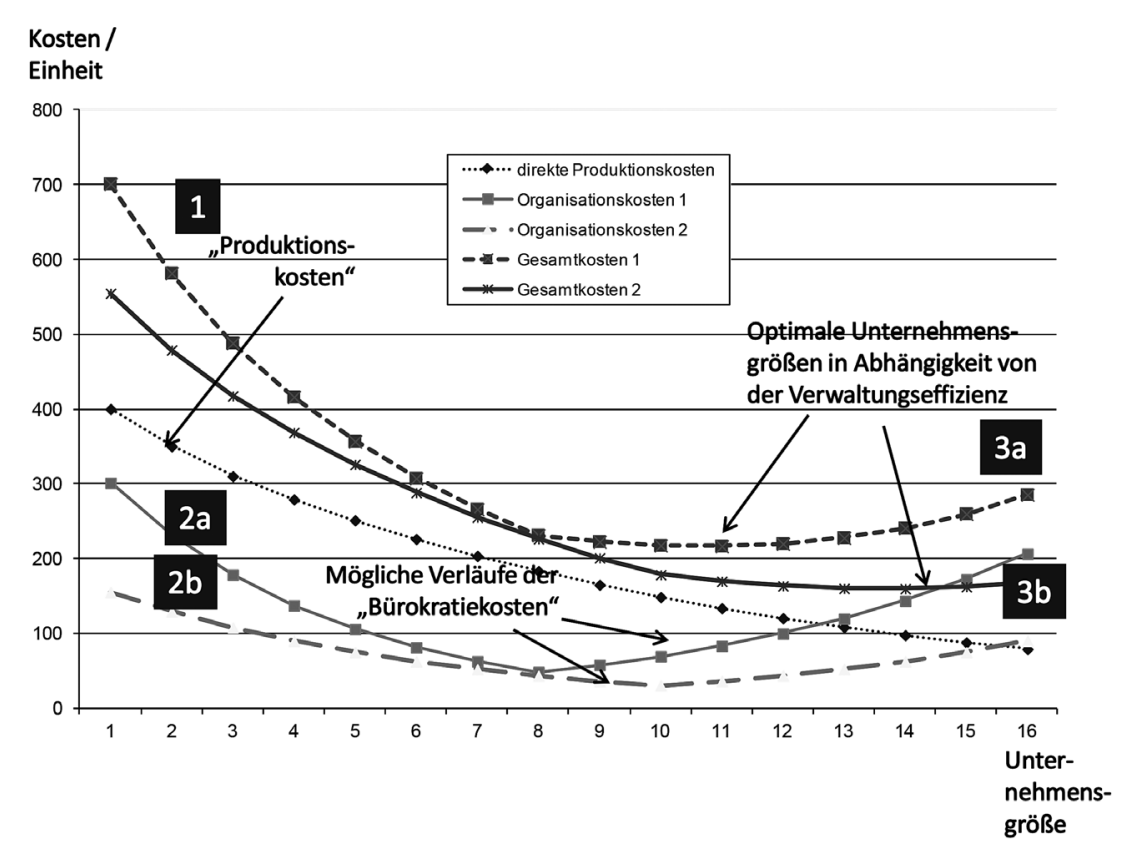

Abb. 1: Der Organisationsaufwand wächst mit der Komplexität und durch die Größe von Verbänden und Unternehmungen zumeist erheblich an.

cen der Führungskräfte und teilweise der Mitarbeiter in den operativen Bereichen.

Hierbei werden zwei idealtypische Verläufe der »Bürokratiekosten" skizziert ( $2 \mathrm{a}$ und $2 \mathrm{~b}$ ), deren Ausprägung von der Effizienz der Verwaltungs- und Führungsorganisation abhängt und die nach einer anfänglichen Abnahme mit steigender Unternehmensgröße wieder ansteigen.

In Addition der beiden Kostenbereiche können die Gesamtkosten je Einheit ermittelt werden, die in diesem einfachen Modell zeigen, dass je nach der Effizienz der Verwaltung sehr unterschiedliche Vorteile aus einer steigenden Betriebsgröße gezogen werden können ( $3 a$ und $3 b$ ). Wenn es nicht gelingt, die Verwaltung der Betriebsgröße entsprechend zu optimieren, können die hier entstehenden Ineffizienzen die möglichen größenbedingten Vorteile der operativen Bereiche schnell neutralisieren, bis hin zu einer Verschlechterung der Gesamtsituation. (Allerdings ist in diesem vereinfachten Modell der mögliche positive Einfluss eines gut organisierten Overheads auf den Verlauf der Produktionsfunktion zunächst noch ausgeblendet.)

Die Problematik einer mit zunehmender Unternehmensgröße überproportional steigenden Overheadgröße ist in der Sozialwirtschaft, in der die meisten Organisationen seit Jahren wachsen und in der zunehmend auch Fusionen und Übernahmen zu verzeichnen sind, nicht selten anzutreffen. Die Leitungs- und Verwaltungsbereiche werden oft nicht konsequent auf eine effiziente Leistungserbringung getrimmt, was einerseits zu hohen Bürokratiekosten, andererseits aber auch dazu führen kann, dass die größenbedingten Vorteile nicht optimal genutzt werden. Daher ist eine zentrale Aufgabe des Managements darin zu sehen, die Verwaltungsaufgaben so zu organisieren, dass eine im Sinne der Gesamtorganisation effiziente Verwaltung aufgebaut wird.

Doch wann ist die Verwaltung oder »die Bürokratie« effizient?

Bei der Beurteilung dieser Frage ist die Prozesseffizienz, die auch die kritische Hinterfragung der einzelnen Aufgaben beinhalten sollte, nur ein Teilaspekt. Durch eine effiziente Organisation dieser Aufgaben können nach unserer Erfahrung bei durchschnittlich aufgestellten Organisationen etwa ein bis zwei Prozent der Gesamtkosten eingespart werden (vgl. Abb. 2).

Viel bedeutsamer ist jedoch die durch die Overheadbereiche zu unterstützende Steuerungseffizienz, durch die das Ergebnis im Umfang zwischen vier bis acht Prozent verbessert werden kann, etwa durch die Unterstützung der operativen Steuerung in den Einrichtungen, das Erschließen größenbedingter Synergien (z. B. Einkauf, Personalpools, Zentralküchen), eine bessere Vermarktung der Dienstleistungen oder eine erfolgreichere Strategieentwicklung. Damit bestimmt die »Bürokratie« durch ihre Leistungen - in Erweiterung des oben beschriebenen vereinfachten Modells - ganz wesentlich auch die Gesamtproduktivität des Unternehmens.

Die konkrete Ausgestaltung der Verwaltungsbereiche wird von einer Reihe von Faktoren beeinflusst. Einen sehr starken Einfluss auf den Aufgabenkatalog, aber auch auf die Ausgestaltung von Organisationen haben externe Anforderungen, die sich beispielsweise aus Gesetzen, vertraglichen Regelungen oder Forderungen von Aufsichtsbehörden ergeben.

Dennoch gibt es auch in diesen Bereichen Gestaltungsfreiräume, die konsequent genutzt werden sollten. So ist öfter festzustellen, dass beispielsweise bei der Auslegung von Buchführungsvorschriften (z. B. Pflege- oder Kranken-

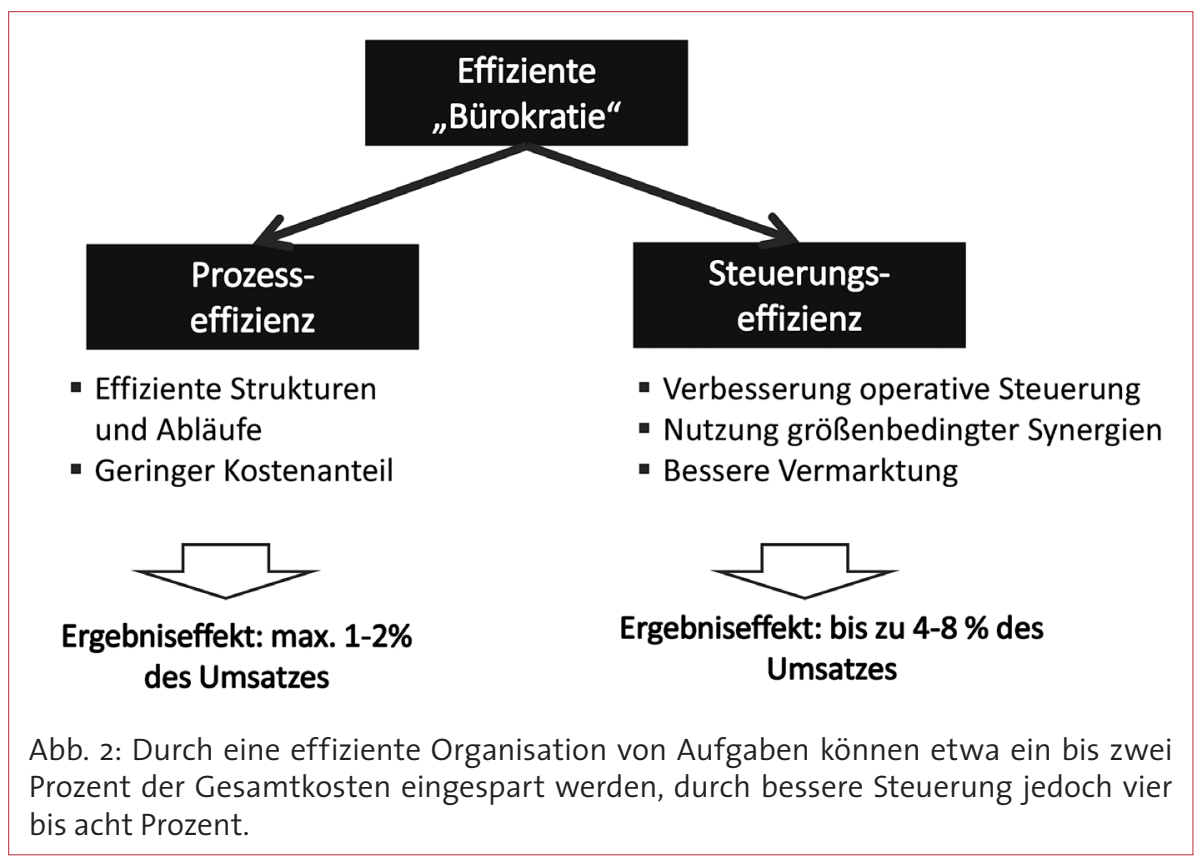


hausbuchführungsverordnung) oder der Ausgestaltung von extern geforderten Systemen des Qualitätsmanagements durch die Organisationen selbst eine höhere Komplexität geschaffen wird, als dies gefordert ist. Aus dieser selbst geschaffenen Komplexität resultieren beispielsweise ein nur aufwendig zu pflegendes Rechnungswesen mit zu vielen Buchungskreisen oder kaum noch beherrschbare Qualitätsmanagementund Dokumentationssysteme, obwohl deutlich schlankere Lösungen zulässig sind. Bei letzterem ist zu erhoffen, dass die kürzlich vorgelegten und im nachfolgenden Beitrag beschriebenen Empfehlungen möglichst bald Einfluss in den Alltag vieler Organisationen finden. de Rolle mehr spielt. So können Doppelarbeiten vermieden werden, unter Nutzung von Schrifterkennungssoftware manuelle Bearbeitungsschritte entfallen und die Prozesse werden durch hinterlegte Workflows transparenter, sicherer und effizienter. Durch erweiterte Möglichkeiten, Arbeitsplätze auch dezentral und damit gegebenenfalls wohnortnah anzubieten, können auch qualifizierte Kräfte besser akquiriert werden.

Generell spielen technische Lösungen eine wesentliche Rolle für die Effizienzsteigerung von Verwaltungsbereichen, sowohl im Sinne der Prozess- als auch der Steuerungseffizienz. Der technische Fortschritt, der Preisverfall in vielen Bereichen (Leitungskosten, mobile Da-

\section{"Die richtige Verwaltung bestimmt} durch ihre Leistungen ganz wesentlich die Gesamtproduktivität des Unternehmens mitu die Möglichkeiten zur verbesserten Steuerung konsequent genutzt werden.

\section{Ganzheitliche Managementperspektive}

Notwendig hierfür ist eine konsequente Nutzung der technischen Möglichkeiten aus einer ganzheitlichen Managementperspektive, die nicht nur den administrativen, sondern auch den Steuerungsaspekten Rechnung trägt. So sollte beispielsweise die elektronische Pflegedokumentation nicht nur der reinen Dokumentation dienen, sondern auch die Basis für ein fachliches Controlling bilden, das jedoch fokussiert zu erfolgen hat. Auch in anderen Bereichen sollten bevorzugt bereits vorhandene Daten aus den eingesetzten Systemen (z. B. Bewohner- und Klientenverwaltung, Dienstplanung, Abrechnung, Finanzbuchhaltung, Personal) genutzt werden, die dann möglichst automatisch in Berichten zusammengeführt werden können, die die Unternehmenssteuerung auf allen Ebenen unterstützen.

Leider ist die Realität heute oft eine andere. Daten liegen in unterschiedlichen Bereichen verteilt vor und es ist die Aufgabe von Verwaltungskräften oder des mittleren Managements die Controller meist händisch mit Daten zu versorgen, die anschließend zu Berichten zusammengeführt werden, die allenfalls der Zentrale aber nicht den Einrichtungen dienen. Die erzeugten Daten helfen damit nur sehr mittelbar der Steuerung, da sie nur verspätet zusammengeführt werden - und nicht selten werden sie nur abgeheftet. Damit werden erhebliche Ressourcen gebunden, ohne einen ausreichenden Mehrwert zu generieren; in einem solchen Fall besteht ein großes Potenzial für »Bürokratieabbau«.

Eine effiziente Verwaltung setzt sich kritisch damit auseinander, welche Daten für wen steuerungsrelevant sind und führt diese zu einem Management-Informations-System zusammen, das alle Ebenen mit den erforderlichen Informationen versorgt. Solch ein ManagementInformations-System wird idealerweise mit einem hohen Selbständigkeitsgrad erstellt, so dass möglichst viele Daten automatisch laufend generiert werden können. So können diverse Kennzahlen insbesondere in den Bereichen »erbrachte Leistungen « und »Personaleinsatz « $\mathrm{zu}$ meist täglich überwacht werden. Der manuelle Aufwand für die Erstellung kann 
eingespart und diese Ressourcen in einen Dialog über die Zahlen umgewidmet werden. Bereits bei Organisationen mittlerer Größe lohnt sich in der Regel die Investition in entsprechende Schnittstellen und Softwaremodule, wenn man nicht nur die Kosteneinsparungen durch eine Steigerung der Prozesseffizienz, sondern auch die zu realisierenden Verbesserungen der Steuerungseffizienz ins Kalkül einbezieht.

Je größer und komplexer die Organisation ist, desto mehr lohnt es sich in der Regel, sich kritisch mit der Overheadorganisation auseinanderzusetzen. Denn sind erst einmal Bereichs- oder Regionalleitungen, Abteilungen, Stabsstellen etc. da, so müssen sie an Besprechungen teilnehmen, in Entscheidungen und Abläufe eingebunden werden, neue Projekte starten, Standards entwickeln und ihre Einhaltung kontrollieren oder eigene Reports erstellen. Solche Strukturen tragen einerseits das Potenzial in sich, die operativen Bereiche wirksam zu unterstützen und Skaleneffekte zu nutzen, andererseits aber auch Prozesse und Entscheidungen schwerfällig und in jedem Fall teurer zu machen. Es ist eine zentrale Managementaufgabe, die Organisation so zu gestalten, dass die Vorteile deutlich überwiegen.
Bei der Neujustierung sowohl der Aufbauorganisation als auch der Ablauforganisation mit dem Ziel der Entbürokratisierung müssen daher stets folgende sieben Leitfragen beachtet werden:

1 Müssen externe Anforderungen tat- sächlich im aktuellen Umfang mit den jetzigen Instrumenten und Abläufen bedient werden?

2 Werden die Strukturen und Pro2. zesse auf die kurz-, mittel- und langfristigen Erfolgsfaktoren des Unternehmens fokussiert und stehen diese in einem gesunden Verhältnis zueinander?

3. Werden die richtigen Prioritäten 3. gesetzt? Sind die intern formulierten Anforderungen an Verwaltungsaufgaben im Sinne der Zielerfüllung des Unternehmens und zur Risikoreduktion angemessen?

4. Stiften die vorhandenen Abläu- fe und Strukturen einen ausreichenden Mehrwert, der die Mehrkosten rechtfertigt? Werden an anderer Stelle tatsächlich Kosten eingespart oder anderweitige Synergien genutzt?
5 Ist der gewählte Prozess (z. B. 5. Kontrollschleifen, Reports) in sich effizient oder vielleicht nur Ausdruck von Unsicherheit, weil an anderer Stelle die Prozesse oder die Strukturen nicht ausreichend gut organisiert oder Funktionen personell nicht adäquat besetzt sind? Kann die Prozessverantwortung an anderer Stelle besser sichergestellt werden?

6. Sind die Abläufe optimal gestaltet? Bestehen Möglichkeiten einer technischen Optimierung, die sich unter Kosten-Nutzen-Effekten rentieren?

7 Können für die intendierten Zwecke - bereits vorhandene Daten genutzt werden? Wie können diese mit möglichst wenig Aufwand bereitgestellt werden?

Die Antworten auf diese Fragen können sich durch Veränderungen der externen Rahmenbedingungen und insbesondere durch neue technische Möglichkeiten im Zeitverlauf deutlich verändern. Daher ist die Auseinandersetzung mit diesen Fragestellungen keine Einmalaufgabe, sondern eine laufende Herausforderung für das Management.

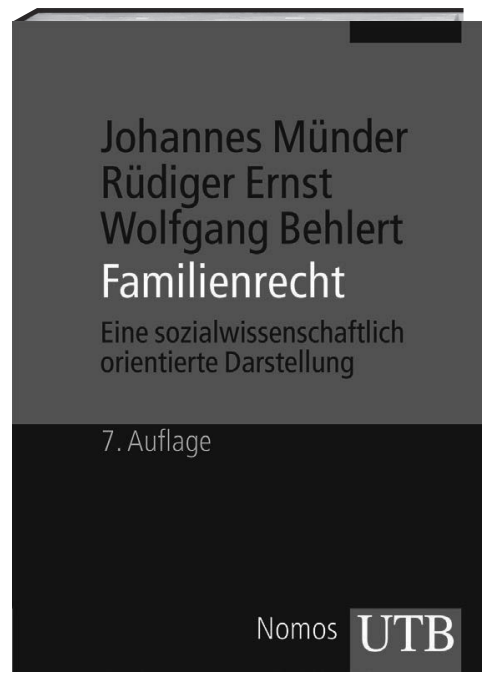

\section{Familienrecht}

Eine sozialwissenschaftlich orientierte Darstellung

Von Prof. Dr. Johannes Münder, RiKG Prof. Dr. Rüdiger Ernst und Prof. Dr. Wolfgang Behlert

7. Auflage 2013, 329 S., brosch., 24,99€

ISBN 978-3-8252-3942-8

www.nomos-shop.de/21056

Das Buch wendet sich an alle, die sich systematisch mit dem Familienrecht befassen. Es stellt das Familienrecht umfassend und sozialwissenschaftlich reflektiert dar. Der Schwerpunkt liegt bei den Regelungen des BGB. Es macht die für das Zusammenleben der Geschlechter sowie für die Sozialisation und Erziehung von Kindern relevanten rechtlichen Regelungen verständlich. 